\title{
POSITION SOLUTION OF COMPACT RADIO SOURCES USING LONG COHERENCE VLBI
}

\author{
J. S. GUBBAY, A. J. LEGG, and D. S. ROBERTSON \\ Australian Defence Scientific Service, Dept. of Supply, Weapons Research Establishment, \\ Salisbury, South Australia
}

\begin{abstract}
Compact components of several radio sources were observed in the course of VLBI observations at $S$-band between NASA-JPL Deep Space Stations located in Australia, South Africa and California, U.S.A., during the southern summer of 1971-72. These stations were equipped with H-maser frequency standards over this period so that the fringe frequency could be determined to better than $1 \mathrm{mHz}$.

The internal consistency of the position solution could be assessed for sources observed at least three times. This varied with source declination from \pm 0 ". 1 at a declination of $45^{\circ}$ to \pm 2.0 near the equator.

The spread in the position solution for P0727-11, a rapid variable at the observing frequency, was also large.

Positions obtained for some other sources have been used to assist in the identification of associated optical objects.
\end{abstract}

During the southern summer of 1971-72, two stations of the NASA-JPL Deep Space Network, DSS 51 at Hartebeestehoek, South Africa and DSS 41 at Island Lagoon, South Australia, co-operated in a series of three interferometer experiments (see Table I). G. Nicolson and P. Harvey of the South African CSIR were responsible for the conduct of the experiments at the South African terminal. This series was followed

TABLE I

Interferometer experiments using independent $\mathrm{H}$-maser frequency standards

\begin{tabular}{|c|c|c|}
\hline Epoch & Western Station designation & Eastern station designation \\
\hline $\begin{array}{rr}357-8 & 1971 \\
21 & 1972 \\
44-5 & 1972\end{array}$ & $\begin{array}{l}\text { DSS 51, Hartebeestehoek near } \\
\text { Johannesburg, South Africa }\end{array}$ & $\begin{array}{l}\text { DSS } 41 \text {, Island Lagoon near } \\
\text { Woomera, South Australia }\end{array}$ \\
\hline $52-3 \quad 1972$ & DSS 41 & DSS 12 , Goldstone, California, U.S.A. \\
\hline
\end{tabular}

within a few days by an experiment between DSS 12 near Goldstone in California and the Australian station, DSS 41. The three stations taking part in experiments across the two intercontinental baselines were equipped with $26 \mathrm{~m}$ antennae, maser front ends and hydrogen maser frequency standards.

Cohen and Shaffer took the results of a survey for compact components using a trans-Pacific baseline between the $64 \mathrm{~m}$ telescope at Goldstone, California and the $26 \mathrm{~m}$ telescope at Tidbinbilla, Australia, to determine the position of compact components. Their position determinations were among the first using VLBI techniques with independent local oscillators. The accuracy of these positions was limited by the stability of the station rubidium frequency standards and by the single parameter atmospheric model adopted. 
The experiments described here were part of a continuing study of the secular behaviour of the $13.1 \mathrm{~cm}$ emission from the compact components of radio sources. They were not designed to yield accurate source positions and station locations. Data was sampled at $48 \mathrm{kbits} \mathrm{s}^{-1}$ at a noise equivalent bandwidth of $14.3 \mathrm{kHz}$. The accuracy of group delay measurement is therefore limited to about 2 microseconds whereas the high stability $\left(1\right.$ part in $\left.10^{14}\right)$ of the frequency standard allows an accuracy of about $20 \mu \mathrm{Hz}$ in the phase-delay rate between the signals at the two stations. Consecutive dual observations, each of $660 \mathrm{~s}$ duration, were made on each source when its elevations from the respective stations were near optimum. However, three runs were made on each of three sources, including 3C 273 and 3C 279 in the first of the series of Southern Hemisphere, inter-continental baseline experiments. The corresponding position error triangles were consistent with a total reading error in the observed Doppler difference frequency of $0.5 \mathrm{mHz}$. Uncertainties in the atmospheric parameters, variations in the length of day and noise contribute to the total error. In addition, the orbital acceleration of the Earth between the respective arrival times at the two stations has not been taken into account.

These three sources were observed àgain during the second southern baseline viewing period. The observations of two of these sources were inter-leaved. Their position solutions however did not correspond with that obtained previously but it was found that the deviation in millihertz from the previous solutions followed a smooth curve suggesting a variable relative drift rate over the second half of the experiment (see Figure 1). Consequently, these results were ignored. The stability requirement of the $S$-band synthesizer for accurate position solutions is near its design limits. The synthesizer was found by Fanselow et al. (1971) to be a likely cause of variable drift. None of the sources observed in the first of this series was viewed in the third experiment.

The trans-Pacific experiment was conducted in conjunction with A.T. Moffet and D. Shaffer of the California Institute of Technology and D. Spitzmesser of the Jet Propulsion Laboratories. The only significant difference in equipment configuration from that adopted for the southern baseline experiments was the substitution of the $S$-band synthesizer by a multiplier chain. Three observations were taken on 3C 273 and four on $3 \mathrm{C} 279$, the last of which was recorded when the source had dropped to an elevation of $5.8 \mathrm{deg}$ from DSS 12 in California. Corrections for the atmosphere are based on a model ionosphere using values of total electron content obtained by F. Hibberd of the University of New England in Northern New South Wales and from the California Institute of Technology using geostationary satellites, and a model troposphere corrected for station altitude. As no ionospheric data was available from South Africa the variation in the ionosphere was inferred from the Australian results by introducing a delay equal to the difference in local time. Bottomside data now available indicate a correction to be applied to the delay. The atmospheric model was developed by K. Lynn of the Weapons Research Establishment. The position solutions for both sources appear to be internally consistent (see Figure 2). For the southern baseline and the trans-Pacific baseline, the separation in right ascension 


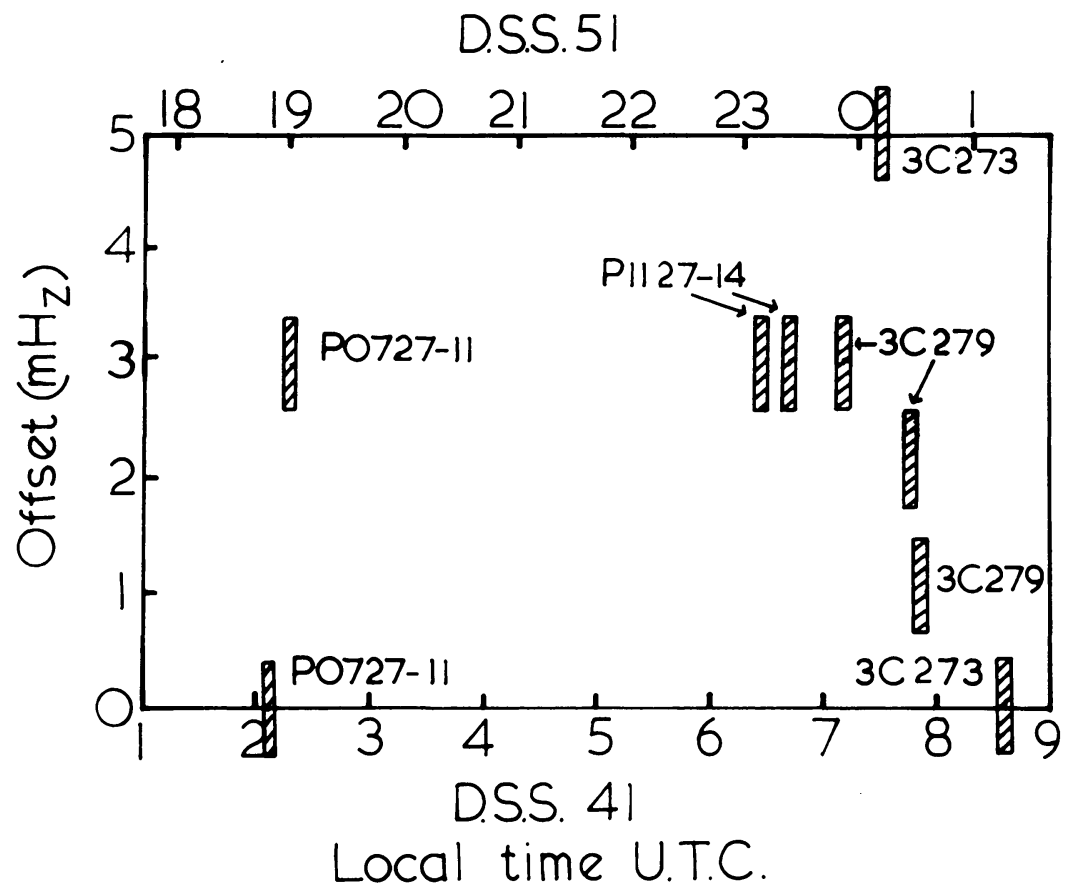

Fig. 1. Relative bias for two trans-Indian Ocean experiments (day 3471971 - day 21 1972).
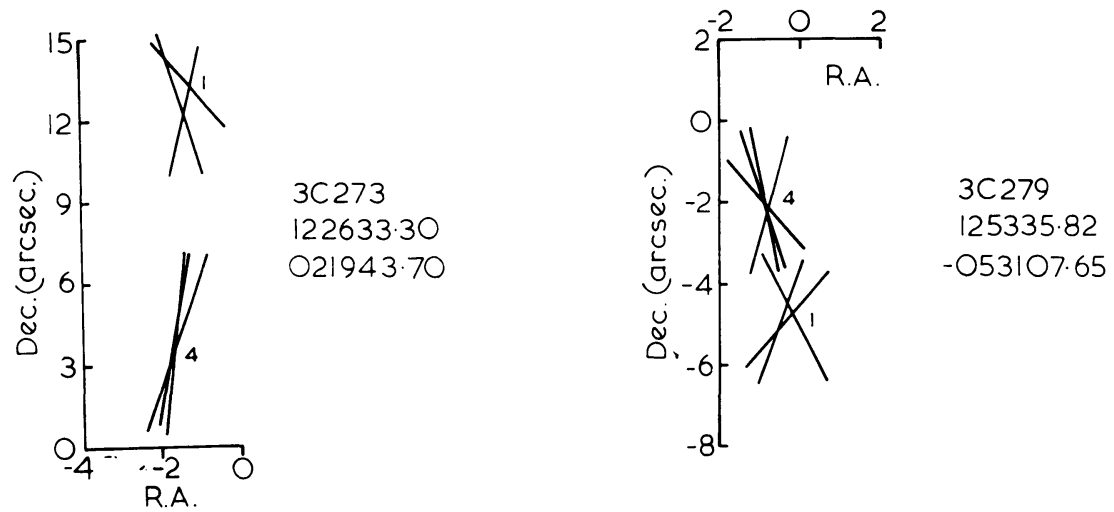

Fig. 2. Source positions using $H$-maser frequency standard (R.A. in units of $s \times 15$ ). 1 - South Africa-Australia 358 1971; 4 - Australia-U.S.A. 531972.

between these two sources are in fair agreement and are consistent with the separation observed by Rogers et al. using VLBI across the United States at a wavelength of $3.8 \mathrm{~cm}$. Agreement with the R.A. of the optical object associated with $3 \mathrm{C} 273$ given by Barbieri et al. (1972) and the R.A. of the optical object associated with 3C 279 is within 0.8 for either baseline. For each source, the difference in declination between 
the two baseline solutions would result from the same relative error in length of baseline or the same difference in relative drift frequency. An adjustment of about $-10 \mathrm{~m}$ to the trans-Pacific baseline and about $-20 \mathrm{~m}$ to the southern baseline would yield good agreement with the source declinations determined by Rogers et al. (1974). However, an error of this magnitude would tend to be reflected in a right ascension error larger than the errors observed for the trans-Pacific baseline, if the station spin distances are correct. An alternative and more likely cause is a relative drift error of $-5 \mathrm{mHz}$ for the trans-Pacific baseline and $-10 \mathrm{mHz}$ for the southern baseline. These figures are consistent with a long term stability figure of 2 parts in $10^{-12}$ for the $S$-band synthesizer. This seems rather high but it should be noted that the relative drift rate appears to have halved for the trans-Pacific experiment where one station used a frequency multiplier instead of the synthesizer.

The Space Research Group at W.R.E. provides source positions to assist optical identifications at Mt Stromlo. Positions of objects beyond the viewing limits of northern interferometers are of particular importance but position determination of a low declination source has also been attempted. In this way, the optical object associated with OV 236, an optical and radio variable, was identified by B. Peterson of Mt Stromlo observatory. With the commissioning of the $64 \mathrm{~m}$ antenna at Tidbinbilla, Australia, a larger number of sources of possible optical interest may be observed.

Three observations were made on 3C 279 by DSS 43, the $64 \mathrm{~m}$ antenna at Tidbinbilla, and DSS 11 in California. The use of Rubidium frequency standards limited the period of phase coherent integration to $60 \mathrm{~s}$ with a consequent increase in random error. However, although different stations were used in this later experiment, values of R.A. of 3C 279 for the two trans-Pacific experiments about 13 months apart are in good agreement. The results from the two trans-Pacific baseline experiments suggest that the true baseline is about 0 ".8 eastwards of the assumed direction, if the compact component coincides with the optical object. The origin of the co-ordinate system for 3C 279 in Figure 2 is at the position for the optical object given by Kristian and Sandage (1970). The difference between the true and assumed directions of the southern baseline appears to be smaller but in the same sense. The sense of the required corrections to the two baselines is opposite to that obtained by Cohen and Shaffer (1971) for the Tidbinbilla-Goldstone baseline involving DSS 42, a $26 \mathrm{~m}$ telescope, less than $300 \mathrm{~m}$ north of DSS 43, the $64 \mathrm{~m}$ telescope at Tidbinbilla and DSS 14 at Goldstone. The difference between the location solution LS 25 and that adopted in this work LS 35 would account for a difference in the correction to the hour angle of the baseline of less than 0 ". 1 . The authors concluded however that the correction obtained then could not be used to improve the station locations.

The equatorial projections of the two baselines, California-Australia-South Africa, span $216^{\circ}$ of longitude. The maximum error in the Hour Angle of the California (DSS 12 or DSS 11) - South Africa (DSS 51) baseline can be deduced from the equatorial projections and errors in hour angle of the other two baselines forming a triangle in the equatorial plane. Thus the hour angle of a source can be determined 
to better than 1.0 , even when only one source is observed, using any of these NASAJPL baselines. The results of all the sources observed in this series will be used to determine the errors in the baselines, the source declinations and the rate offsets for each experiment.

\section{References}

Barbieri, C., Capaccioli, M., Ganz, R., and Pinto, G. : 1972, Astron. J. 77, 444.

Cohen, M. H. and Shaffer, D. B.: 1971, Astron. J. 76, 91.

Fanselow, J. L., MacDoran, P. F., Thomas, J. B., Williams, J. G., Finnie, C. J., Sato, T., Skjerve, L., and Spitzmesser, D. J.: 1971, JPL Tech. Rept. 32-1526. Vol. V, pp. 45-57.

Kristian, J. and Sandage, A.: 1970, Astrophys. J. 162, 391.

Rogers, A. E. E., Counselman, C. C., III, Hinteregger, H. F., Knight, C. A., Robertson, D. S., Shapiro, I. I., Shitney, A. R., and Clark, T. A.: 1974, submitted to Astrophys. J. Letters. 